\title{
On Comparing Feature Reduction Techniques for Accuracy Improvement of the k-NN Classification
}

\author{
Sak Phoemhansa*, Keerachart Suksut, Phaichayon Kongchai, \\ Rattaphong Sutamma, Nittaya Kerdprasop, Kittisak Kerdprasop \\ School of Computer Engineering, Institute of Engineering, \\ Suranaree University of Technology, Nakhon Ratchasima, 30000, Thailand \\ *Corresponding Author: sak_jeek@ hotmail.com
}

\begin{abstract}
The aim of this paper is to perform a comparative study of feature reduction techniques that are most appropriate for the classification with k-nearest neighbor and tested with medical data. Medical data are normally high-dimensional in their nature. Their high dimensionality property can affect performance of the classification process. In this work, we perform various feature reduction techniques implemented with Matlab to decrease dimensions of data before the knearest neighbor classification step. From the experimented results, we found that best performance is obtained from using the PCA algorithm to reduce features of data. The comparison in terms of accuracy turns out that PCA and ROC feature reduction techniques can improve the classification prediction, whereas the t-test feature reduction has very limited effect over the classification accuracy.
\end{abstract}

Keywords: data mining, dimension reduction, k-NN.

\section{Introduction}

Techniques to reduce the feature of data is one step in the data preprocessing it have important with pattern recognition and high-dimension data. This techniques can be remove attributes without affect the performance of algorithm. Because some attributes may affect the performance of algorithm. That is attributes may not be related to class label of data. We use feature reduction techniques for remove irrelevant attributes.

k-Nearest Neighbor is most popular in the fields of pattern recognition and machine learning. The algorithm is based supervised learning. It is finding nearest of $\mathrm{k}$ data.
In this paper, we are used feature reduction techniques as PCA, t-test and ROC in first step for improve effect classification with k-nearest neighbor algorithm. The experimental result to compare the accuracy from experiments with each techniques, error and number of attributes of classify. As medical data is a very high number of attributes to suitable test data in evaluation.

\section{Related works}

Feature reduction is important in data preprocessing and widely used because it can extract important part. Related work use of this techniques as Mauricio Villegas and Roberto Paredes $^{(1)}$ used dimension reduction techniques with LDPP for test data from UCI and estimation error of k-NN, the result concluded that use LDPP can optimize k-NN. Phattrawut Sangsiri et $\mathrm{al}^{(2)}$. compare the performance of dimension reduction with PCA and BFSF then the artificial neural network to predict the cancer data, the result BSFS techniques appropriate to input neural network. Deqing Wang et al. ${ }^{(3)}$ use t-test algorithm to feature selection, then use k-NN and SVM test performance with text data. D.A. Adeniyi et $\mathrm{al}^{(4)}$. they present automatic web usage data mining with k-nearest neighbor. The classification method has been trained to be used on-line and in real-time to identify clients/visitors click stream data. Yi-Hung Kung et $\mathrm{al}^{(5)}$. they find that the asymptotically optimal linear combination of nearest neighbors for density estimation is just the last term of the linear combination. Thananan Prasartvit et $\mathrm{al}^{(6)}$. proposed improved method for data dimensionality reduction called ABC-kNN. The method uses the wrapper technique for classification. 


\section{Feature Reduction and Classification}

\subsection{Feature Reduction Techniques}

(a) Principal Component Analysis (PCA)

The Principal Component Analysis is used reduce features for high-dimension data and the most popular multivariate statistical technique. It a significant linear component analysis $^{(7)}$. If any component is more important to selected and number of components is less than or equal the number of original components. The algorithm consist eigenvectors and eigenvalues.

Fig. 1 shown plotting the eigenvectors of covariance matrix (blue line) and point of all data before reduce features. And when used PCA to reduce features of data in Fig. 2.

(b) T-test

The T-test ${ }^{(8)}$ algorithm is statistical test to compare the difference of the mean values in two group. By compute $t_{0}$ value of two features from equation (1). Compute degree of freedom $(d f)$ value and define rho $(\alpha)$. Correlate $d f$ value and rho with t-table is $t$ value. Then consider that reduce features from checking $t_{0}$ and $t$ value that difference or equal. T-test is based on a normal data distribution.

There are three types.

- One sample t-test

- Independent sample t-test

- $\quad$ Paired sample t-test

$$
t_{0}=\frac{\overline{X_{B}}-\overline{X_{A}}}{\sqrt{S_{p}^{2}\left(\frac{1}{n_{B}}+\frac{1}{n_{B}}\right)}}
$$

Where $X_{A}$ is mean value of $A$ sample, $X_{B}$ is mean value of $B$ sample, $S_{p}^{2}$ is variance of $p$ sample and $n_{B}$ is number sample of $B$ sample.

(c) The Receiver Operating Characteristic Curve (ROC)

The ROC is measure of the class-discrimination by look at plotting the relationship between the true positive rate and false positive rate. The method is measuring overlap of the data distribution two group.

Fig. 3 shown plotting graph of ROC curve by plot the corresponding AUC values of data before reduce features. AUC value of data is less than 0 which diagonal line is equal 0 . And when reduced features with ROC algorithm the AUC value increase is more than 0 in Fig. 4.

For example, Table 1 shown sample the data consist 4 instance, 3 features and class label which each instance is numeric data. Then bring the data to preprocessing data step with using ROC algorithm for reduce features of data. When

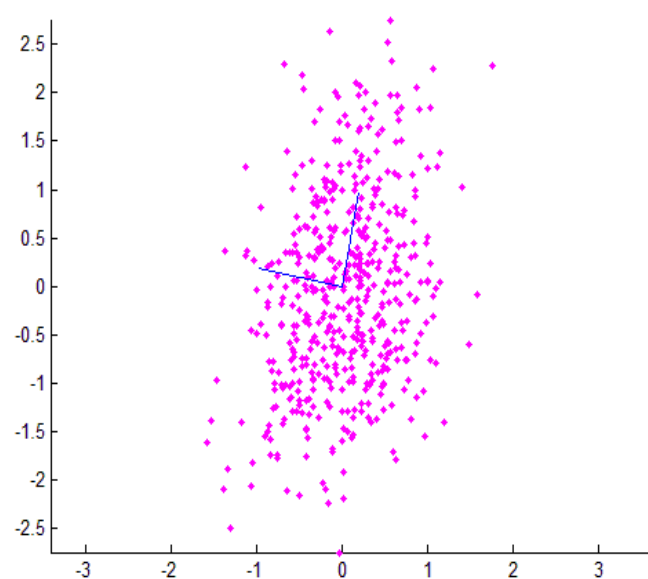

Fig. 1. The sample of data in experiment with PCA.

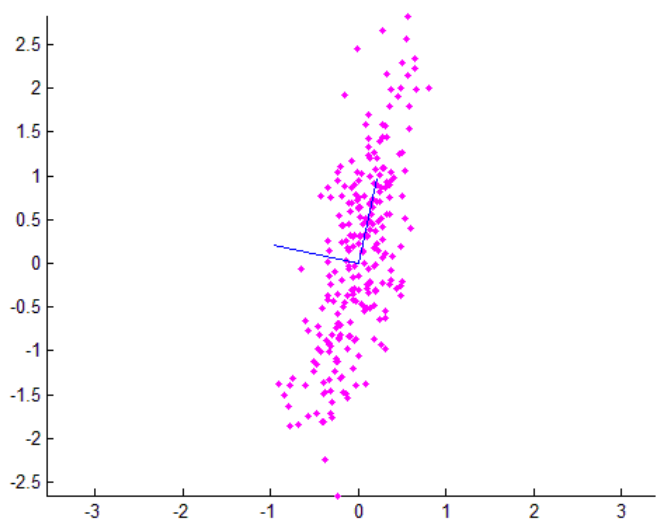

Fig. 2. The sample of data reduced with PCA.

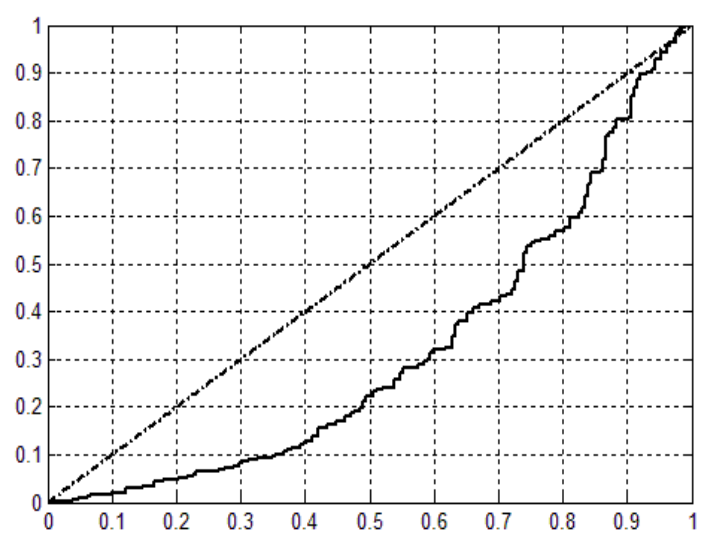

Fig. 3. The sample of plot data with ROC Curve.

reduced features the number of features remain is 2 features in Table 2. The features in Table 2 is important features to class label of data.

\section{2 k-Nearest Neighbor Classification Algorithm}

The algorithm is based on supervised learning ${ }^{(9)}$. It find nearest $\mathrm{k}$ sample from training data and identified class of 
Table 1. The sample of data in experiment.

\begin{tabular}{|c|c|c|c|}
\hline $\mathrm{X}$ & $\mathrm{Y}$ & $\mathrm{Z}$ & Class \\
\hline 52 & 63 & 15 & 1 \\
\hline 48 & 79 & 24 & 1 \\
\hline 94 & 88 & 12 & 2 \\
\hline 36 & 52 & 19 & 2 \\
\hline
\end{tabular}

Table 2. The sample of data reduced feature.

\begin{tabular}{|c|c|c|}
\hline $\mathrm{X}$ & $\mathrm{Y}$ & Class \\
\hline 52 & 63 & 1 \\
\hline 48 & 79 & 1 \\
\hline 94 & 88 & 2 \\
\hline 36 & 52 & 2 \\
\hline
\end{tabular}

new data when new data appears.

Fig. 5 shown the sample of k-nearest neighbor classification. $\mathrm{N}$ denotes new data. It find class of new data $\mathrm{N}$. In solid line circle is $\mathrm{k}$ sample nearest $\mathrm{N}$ (when $\mathrm{k}=5$ ). It is assigned class with similar square data because there are 3 square data is mostly data.

The following step:

- Choose a value for $\mathrm{k}$.

- Compute the distance between consider data and training data.

- $\quad$ Consider nearest data number of $\mathrm{k}$.

- Assign classes the data.

\section{Experimentation and Results}

\subsection{Dataset}

The dataset in experiment is dataset from $\mathrm{UCI}^{(10)}$. Which used 5 dataset as Breast Cancer Wisconsin, Parkinsons, Spect, Pima, Lungcancer. The Dataset is high-dimension and numeric data. The numbers features of each dataset shows in Table 3.

\subsection{A Comparative Study}

Table 3. The numbers of feature.

\begin{tabular}{|l|c|}
\hline \multicolumn{1}{|c|}{ data } & \#Features \\
\hline breast_cancer_wisconsin & 30 \\
\hline parkinsons & 22 \\
\hline spect & 44 \\
\hline pima & 8 \\
\hline lungcancer & 56 \\
\hline
\end{tabular}

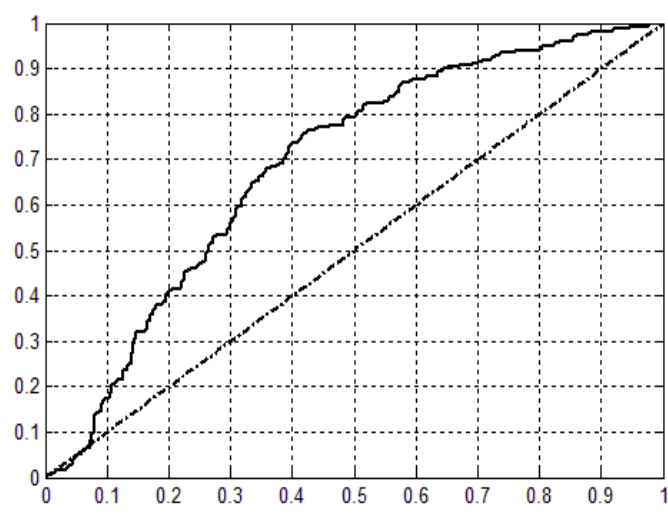

Fig. 4. The sample of plot ROC with reduced features.

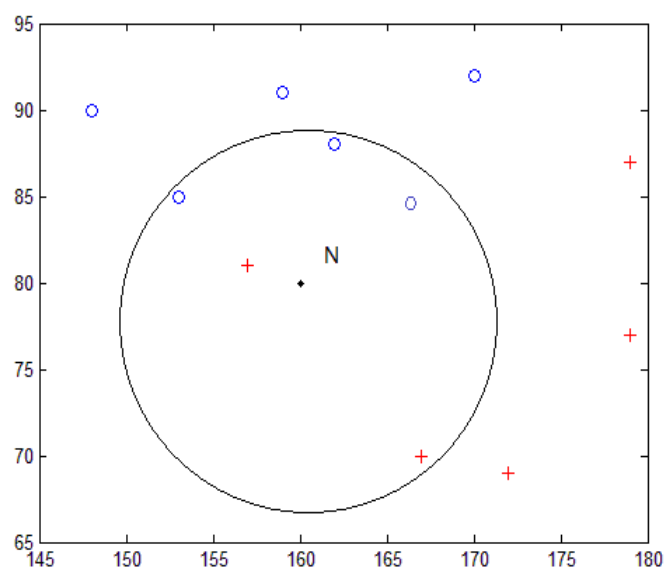

Fig. 5. The sample of k-NN classification.

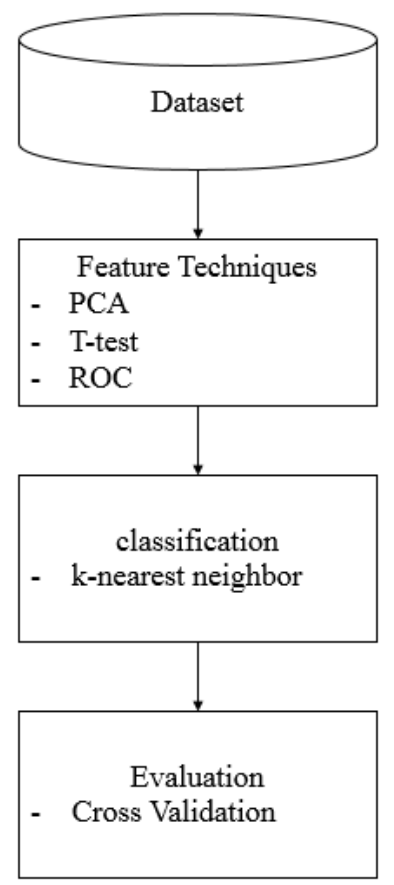

Fig. 6. The framework of technique. 
Fig. 6 shown all step of the working. Firstly prepare dataset. Second use dimension reduction techniques with existing dataset. Next classify with k-nearest neighbor. And then evaluate with cross validation.

\subsection{Experimental Results}

The method implement with Matlab in experiment. Firstly, we are bring dataset to classify with k-nearest neighbor for the performance of classification on each dataset. The results shown in Table 4.

\subsubsection{Classification Results over full-feature data}

Table 4. The result of k-NN classifier without any application of feature reduction.

\begin{tabular}{|l|c|c|c|}
\hline \multicolumn{1}{|c|}{ data } & \#features & err & acc \\
\hline breast_cancer_wisconsin & 30 & 0.095 & 0.905 \\
\hline parkinsons & 22 & 0.1681 & 0.8319 \\
\hline spect & 44 & 0.3357 & 0.6643 \\
\hline pima & 8 & 0.2917 & 0.7083 \\
\hline lungcancer & 56 & 0.4667 & 0.5333 \\
\hline
\end{tabular}

Table 4 shown the error values and accuracy values of classified and \#features is the number of features used in classified. From the results is obtained the best accuracy is Breast Cancer Wisconsin as 0.905 and number of features to used is 30 .

Next, we are used dimension reduction techniques to reduce features of each dataset for improve performance of classifying. The method use in prepare data before classify with k-nearest neighbor.

\subsubsection{Classification Results with PCA Feature Reduction Technique}

Table 5. The classification results with PCA feature reduction.

\begin{tabular}{|l|c|c|c|}
\hline \multicolumn{1}{|c|}{ data } & \#features & err & acc \\
\hline breast_cancer_wisconsin & 18 & 0.0915 & 0.9085 \\
\hline parkinsons & 15 & 0.1648 & 0.8352 \\
\hline spect & 24 & 0.201 & 0.799 \\
\hline pima & 2 & 0.2435 & 0.7565 \\
\hline lungcancer & 30 & 0.3333 & 0.6667 \\
\hline
\end{tabular}

Table 5 shown the error values and accuracy values of classified and \#features is the number of features reduced with PCA algorithm. From using PCA the number features of data less than the number of features in Table 4 and increases accuracy values.

\subsubsection{Classification Results with T-test Feature Reduction Technique}

- $\quad$ Define rho $=0.0001$

Table 6. The classification results with T-test feature reduction $($ rho parameter $=0.0001)$.

\begin{tabular}{|l|c|c|c|}
\hline \multicolumn{1}{|c|}{ data } & \#features & err & acc \\
\hline breast_cancer_wisconsin & 30 & 0.095 & 0.905 \\
\hline parkinsons & 20 & 0.1737 & 0.8263 \\
\hline spect & 44 & 0.3357 & 0.6643 \\
\hline pima & 8 & 0.2917 & 0.7083 \\
\hline lungcancer & 12 & 0.4333 & 0.5667 \\
\hline
\end{tabular}

Table 6 shown the error values and accuracy values of classified and \#features is the number of features reduced with t-test algorithm and defined parameter rho is 0.0001 .

- $\quad$ Define rho $=0.05$

Table 7. The classification results with T-test feature reduction (rho parameter $=0.05)$

\begin{tabular}{|l|c|c|c|}
\hline \multicolumn{1}{|c|}{ data } & \#features & err & acc \\
\hline breast_cancer_wisconsin & 30 & 0.095 & 0.905 \\
\hline parkinsons & 20 & 0.1737 & 0.8263 \\
\hline spect & 44 & 0.3357 & 0.6643 \\
\hline pima & 8 & 0.2917 & 0.7083 \\
\hline lungcancer & 20 & 0.2833 & 0.5982 \\
\hline
\end{tabular}

Table 7 shown the error values and accuracy values of classified and \#features is the number of features reduced with t-test algorithm and defined parameter rho is 0.05 .

From Table 6 and 7, the results is similar but it difference at number of features of lungcancer dataset and accuracy values from defined difference rho value. Which rho value is significance level (1- $\alpha$ ) for hypothesis test. Generally used rho value is 0.05 .

\subsubsection{Classification Results with ROC Feature Reduction Techniques}

Table 8. The classification results with ROC feature reduction.

\begin{tabular}{|l|c|c|c|}
\hline \multicolumn{1}{|c|}{ data } & \#features & err & acc \\
\hline breast_cancer_wisconsin & 15 & 0.0485 & 0.9515 \\
\hline parkinsons & 18 & 0.1735 & 0.8265 \\
\hline spect & 24 & 0.4088 & 0.5992 \\
\hline pima & 5 & 0.2787 & 0.7213 \\
\hline lungcancer & & & \\
\hline
\end{tabular}

Table 8 shown the error values and accuracy values of classified and \#features is the number of features reduced 


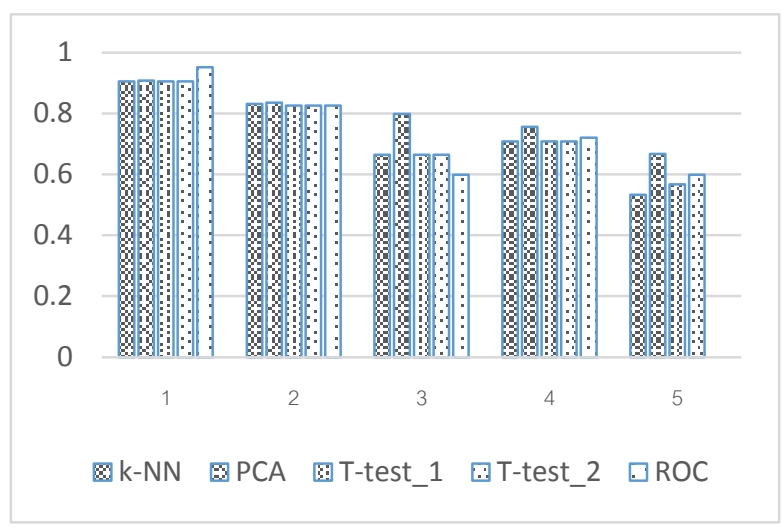

Fig. 7. Chart shown the accuracy of algorithm.

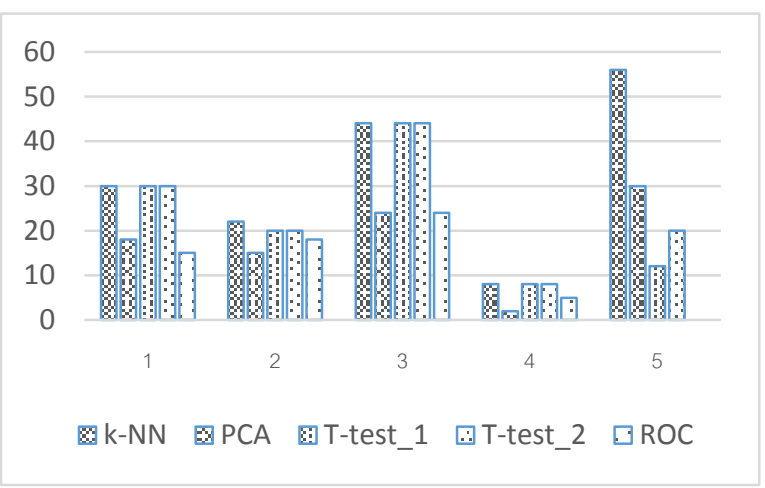

Fig. 8. Chart shown reduced number of features.

with ROC algorithm. It has a blank in Table 8 is not value.

From the experimental results, we use k-nearest neighbor to classify and the result is obtained in Table 4. Then reduce features with various techniques for optimize classification. In experiment, we are compare results from using the dimension reduction techniques as ROC algorithm reduced features. As Spect dataset has existing features is 44 reduced features with ROC algorithm to features remaining is 24 and better performance of classification.

Fig. 7 and 8 shown chart conclude from all experimental results table by number 1 is Breast Cancer Wisconsin dataset, 2 is Parkinsons, 3 is Spect, 4 is Pima, 5 is Lungcancer. Fig. 7 is chart shown comparison accuracy values of each method. Firstly Classification with k-NN the number of features equal original dataset then used feature reduction techniques as PCA, T-test (T-test_1 is used rho 0.0001 and T-test_2 is used rho 0.05), Roc to reduced features. From Fig. 7 shown that using PCA can improve accuracy values of classification greater than or equal to other techniques. Fig. 8 is chart shown comparison the number of features. The k-NN used all features in classification and reduced features with feature reduction techniques. From Fig. 8 shown that using PCA can reduced features about half of features original dataset but not affect to performance of classification and also improve the performance of classification.

From compared results of using feature reduction techniques the best of techniques is PCA algorithm in reduce features of data. The PCA algorithm can reduced irrelevant features and optimize accuracy values. The experiment show that PCA can improve performance of mostly dataset. Which it can improve to the best of classifying on four dataset is about $80 \%$ from all dataset and rest dataset is Breast Cancer Wisconsin improved with ROC algorithm is about $20 \%$.

\section{Conclusions}

This paper presents a performance comparison of feature reduction techniques to be applies prior to the classification with k-nearest neighbor. We used PCA, t-test and ROC algorithms to reduce features in data preparation step. The performance comparative result is that using PCA algorithm to reduce features can increase accuracy values and reduce the most significant number of features. PCA algorithm can improve accuracy in most dataset: Breast Cancer Wisconsin from 0.925 to 0.9085 , Parkinsons from 0.8319 to 0.8352 , Spect from 0.6643 to 0.7990 , Pima from 0.7083 to 0.7565 , Lungcancer from 0.5333 to 0.6667 . We are planning to improve PCA-based feature reduction to be more appropriate for medical diagnosis in terms of model's understandability.

\section{References}

(1) Mauricio Villegas, and Roberto Paredes : "Dimensionality reduction by minimizing nearestneighbor classification error", Pattern Recognition Letters, Vol. 32, issue 4, pp. 633-639, 2011

(2) Phattrawut Sangsiri, Sreemaj Nawichian, and pyung Meesuj : "A Comparing Efficiency of Dimension Reduction for Artificial Neural Network Between Backward Stepwise Feature Selection and Principal Component Analysis for Predicting Cancer Data Set", NCCIT, pp. 851-858, 2009

(3) Deqing Wang, Hui Zhang, Rui Liu, Weifeng Lv, and Datao Wang : "t-Test feature selection approach based on term frequency for text categorization", Pattern Recognition Letters, Vol. 45, pp. 1-10, 2014

(4) D.A. Adeniyi, Z. Wai, and Y. Yongquan, "Automated web usage data mining and recommendation system using K-Nearest Neighbor (KNN) classification 
method", Applied Computing and Informatics, 2014

(5) Yi-Hung Kung, Pei-Sheng Lina, and Cheng-Hsiung Kao, "An optimal k-nearest neighbor for density estimation", Statistics \& Probability Letters, Vol. 82, pp.1786-1791, 2012

(6) Thananan Prasartvit, Anan Banharnsakun, Boonserm Kaewkamnerdpong, and Tiranee Achalakul, "Reducing bioinformatics data dimension with ABC-kNN", Neurocomputing, Vol. 116, pp.367-381, 2013

(7) Mohamed Morchid, Richard Dufour, Pierre-Michel Bousquet, Georges Linare, and Juan-Michel TorresMoreno : "Feature selection using Principal Component Analysis for massive retweet detection", Pattern Recognition Letters, Vol. 49, pp. 33-39, 2014

(8) Prachong Satam : "T-test", Available on : https://www.gotoknow.org/posts/399528

(9) Mehmet Aci, Cigdam Inan, and Mutlu Avci : "A hybrid classification method of k-nearest neighbor, Bayesian method and genetic algorithm", Expert Systems with Applications, Vol. 37, issue 7, pp. 5061-5067, 2010

(10) UCI Machine Learning Repository : Data set, Available on :https://archive.ics.uci.edu/ml/datasets.html 